\title{
HABILIDADES DE CODIFICACION Y DESCODIFICACION DE TABLAS Y GRAFICOS ESTADISTICOS: UN ESTUDIO COMPARATIVO EN PROFESORES Y ALUMNOS DE PEDAGOGIA EN ENSEÑANZA BASICA ${ }^{1}$
}

\author{
Francisco Rodríguez Alveal* \\ Pedro Rodrigo Sandoval Rubilar**
}

*Magíster en Bioestadística. Universidad del Bío-Bío, Chillan, Chile. E-mail: frodriguez@ubiobio.cl

***Doctor en Ciencias de la Educación. Universidad del Bío-Bío, Chillan, Chile. E-mail: psandoval@ubiobio.cl

Resumen: El artículo presenta los resultados de la evaluación realizada a profesores de educación general básica en ejercicio y estudiantes en formación de la carrera de Pedagogía en Educación General Básica. Corresponde a una evaluación de tipo descriptiva y comparativa de corte transversal, a una población compuesta por 47 profesores en ejercicio y 44 alumnos en Formación Inicial Docente (FID) a quienes se les aplicó un instrumento debidamente validado por experto, con alfa de Cronbach $\alpha=0,75$ y un índice de validez de contenido de 0,66 . En el análisis de la información se utilizaron métodos descriptivos tales como promedio, desviaciones estándar y frecuencia porcentual. Los resultados evidencian que en general, los Profesores en ejercicio y alumnos en FID realizan procesos de descodificación por debajo de lo esperado y, según la clasificación de Curcio, se ubicarían en el nivel uno de los cuatros postulados, es decir presentan las habilidades básicas o iniciales asociadas a la descodificación y análisis de los datos.

Palabras claves: Representaciones gráficas. Formación de profesores. Evaluación de habilidades estadísticas.

\author{
STATISTICAL TABLES AND GRAPHS CODIFICATION AND \\ DECODIFICATION ABILITIES: A COMPARATIVE STUDY WITH PEDAGOGY \\ TEACHERS AND STUDENTS OF BASIC ELEMENTARY EDUCATION
}

Abstract: The article presents results obtained from a test application to 47 in-service and 44 prospective primary school teachers. Both groups responded a descriptive comparative cross-assessment test, validated by expert, with a Cronbach alpha reliability of 0.75 and a content validity index of 0.66 . For data processing and analysis, descriptive methods such as average, standard deviation and percentage frequency were used. Results show that, in general, both in-service and prospective primary school teachers apply decoding processes below expected levels, which means that, according to Curcio's categorization, subjects would belong to level one. In other words, they tend to have basic or initial skills associated to decoding and analysis of information.

Key words: Graphs representations. Teacher initial preparation. Statistical skills assessment.

\section{ANTECEDENTES}

Una de las características de la sociedad actual es la gran cantidad de información que circula por la red, libros, periódicos e informes necesarios para la toma de decisión en los más diversos ámbitos productivos mundiales. Mucha

1 El presente estudio se deriva del Proyecto UBB código. 103823 1/1 Formación Inicial Docente. 
de esta información corresponde a codificaciones de datos con la cual se pretende ilustrar realidades mediante un lenguaje matemático, fundamentarlas o proyectarlas para el conocimiento o comprensión de diferentes fenómenos materiales, sociales, naturales, entre otros.

Es en este escenario es donde el modelamiento de los fenómenos a través de datos y representaciones graficas adquiera vital importancia para comprender en mundo actual. Por ello es que la estadística es hoy parte del currículo de las matemáticas desde la educación primaria hasta la secundaria en muchos países, debido a su utilidad en la vida diaria, como también el rol que juega en otras disciplinas, la necesidad del conociendo estocástico básico para muchas profesiones y su importancia para el razonamiento crítico (BATANERO, 2001).

En particular las representaciones gráficas tienen un papel esencial en la organización, descripción y análisis de datos como en la representaciones de los distintos fenómenos de la vida moderna, debido a que dicha área del saber resulta ser un instrumento de transnumeración (formas básicas de razonamiento estadístico) que consiste en obtener una nueva información o al cambiar de un sistema de representación a otro (WILD et al, 1999).

En este contexto, el Ministerio de Educación de Chile (MINEDUC), durante el año 2009, aprobó un nuevo marco curricular para el Sistema Escolar chileno, siendo uno de los aspectos más relevantes el hecho que los contenidos de Modelamiento de Datos (estadísticas) son incorporados desde el primer año básico en el sector de Matemática ${ }^{2}$, con la finalidad de desarrollar el razonamiento probabilístico, modelamiento de datos e interpretación de ellos, habilidades propias de la Estadística (CHILE, 2009a).

Si bien está incorporación de contenidos propuesta en el nuevo marco curricular es necesaria y relevante, ello supone condiciones básicas para su aplicación en el aula, así pues el conocimiento, dominio y competencias asociadas a la estadística por parte de los profesores resulta clave. Según Batanero (2001) y Arteaga (2010), puesto que habitualmente los profesores suponen que la elaboración de tablas y gráficos es muy sencilla y dedican poco tiempo a su enseñanza. Sin embargo, elaborar una tabla de frecuencia o un gráfico supone, una primera reducción estadística compleja, puesto que se trata de transformar los valores originales (de un fenómeno o hecho) de la realidad en representaciones de datos o valores individuales y/o grupales.

Por otro lado, según los resultados de $\operatorname{PISA}^{3}$ (2000) que mide la capacidad para aplicar y relacionar conocimientos y habilidades en Lectura, Matemáticas

2 Corresponde al inicio de la formación escolar obligatoria en Chile, a la edad de 6 años.

3 Evaluation Program for International Student Assesment (PISA) 
y Ciencias, señala que en el área de Matemática, los resultados obtenidos por los estudiantes chilenos los ubican en el nivel de habilidades más básicas. En términos operativos, esto significa que pueden completar procedimientos de un solo paso, como reproducir procesos matemáticos básicos o hacer cálculos simples.

Otros estudios señalan que las habilidades asociadas a geometría, datos y azar son descendidas en los estudiantes en Formación Inicial Docente, también señalan, que las habilidades propias de la Comprensión Lectora tales como codificación, descodificación, re-organización de información, entre otras, pueden tener un grado de correlación con los procesos de razonamiento y resolución de problemas matemáticos (RUBILAR et al, 2010).

En otras palabras, las habilidades asociadas a la resolución de problemas matemáticos, específicamente, en los referido a representaciones gráficas de datos tendrían alguna relación con los procesos de comprensión lectora, toda vez que la lectura supone procesos de descodificación e interpretación de grafema (letras o números), y la interpretación de gráficos supone procesos de descodificación y codificación de información representada en un grafema (gráfico).

En este contexto resulta vital no sólo saber si los estudiantes son capaces de interpretar correctamente la información a partir de una representación gráfica, si no además, saber cuáles son sus dificultades para comprender dicha información en los procesos de lectura (descodificación), análisis e interpretación, así cómo la representación de fenómenos reales (codificación).

Siguiendo la idea anterior, la enseñanza de la estadística, generalmente se basa en graficar información cuantitativa vía histogramas sin dedicar tiempo para "hacer hablar" la representación gráfica y dar cuenta del mensaje que guardan los datos detrás de la representación gráfica, siendo lo que más importa es la representación de la realidad representada más que el mero calculo numérico (GAL, 2002).

En este contexto, se espera que los docentes puedan enseñar a representar (codificar), analizar e interpretar (decodificar) información gráfica a sus estudiantes que serán los futuros ciudadanos del mundo. En otras palabras, como sostiene Mooney (2002), se debe aprender una racionalidad estadística que permita a los individuos descodificar, describir, analizar, codificar e interpreta la información representada gráficamente.

Ahora bien, cómo sostienen algunos autores, nadie puede enseñar lo que no conoce o domina (HERNÁNDEZ; SANCHO, 1996; RODRÍGUEZ, 1995), resulta relevante estudiar el dominio de las habilidades de descodificar, interpretar e inferir la información representada gráficamente, por parte de los profesores 
en ejercicio y en formación, pues ellos son y serán los responsables de desarrollar dichas habilidades a las actuales y futuras generaciones. También resulta relevante indagar en el proceso inverso, es decir, cómo de un fenómeno real o representado ejercen su habilidad de codificación para representarla.

En otras palabras, y asociados a la habilidades de comprensión lectora, y siguiendo la clasificación de Curcio (1989), resulta relevante estudiar cómo leen entre, dentro y más allá de los datos representados y la habilidad para codificar información en una representación gráfica los Docentes en Ejercicio y en Formación Inicial Docente.

\section{OBJETIVO GENERAL}

Comparar el nivel de habilidades de Descodificación e Interpretación de información gráfica, como de los argumentos explícitos para justificar los procesos de Codificación de información representada gráficamente, entre los profesores de Enseñanza General Básica en ejercicio y Estudiantes en Formación Inicial Docente en Pedagogía en Educación General Básica.

\section{Objetivos Específicos}

1.- tipificar las habilidades de Descodificación e Interpretación de Representaciones en Tablas y Gráficos, de los profesores de Enseñanza Básica en ejercicio y Estudiantes en Formación Inicial Docente en Pedagogía en Educación General Básica;

2.- clasificar las habilidades de Codificación en Representaciones Gráficas, de los profesores de Enseñanza Básica en ejercicio y Estudiantes en Formación Inicial Docente en Pedagogía en Educación General Básica;

3.- codificar los argumentos explícitos para justificar los procesos de Codificación de información, para ser Representada Gráficamente, que realizan los profesores de Enseñanza Básica en ejercicio y Estudiantes en Formación Inicial Docente en Pedagogía en Educación General Básica;

4.- comparar el desempeño de las habilidades de Descodificación e Interpretación de Representaciones gráficas y las razones o argumentos explicitados, dados por los Profesores de Enseñanza Básica en ejercicio y Estudiantes en Formación Inicial Docente en Pedagogía en Educación General Básica, en el proceso de Codificación de información para ser Representada Gráficamente; 


\section{Preguntas Orientadoras del estudio:}

- ¿existe diferencias en el nivel de habilidades que tienen los profesores en ejercicio y en Formación Inicial Docente, para leer (descodificar), analizar e interpretar datos o información;

- ¿existe diferencias en el nivel de habilidades que tienen los profesores en ejercicio y en Formación Inicial Docente, para codificar datos o información en representaciones gráficas?;

- al codificar datos o información en representaciones gráficas, que saberes o conocimientos emplean o aplican para ello.

\section{MARCO TEÓRICO}

Las representaciones gráficas aparecen en la literatura a partir de 1789, siendo el ingeniero y economista escocés Willian Playfair el que introdujo las tablas de distribución de frecuencias, los gráficos de barras simples y gráficos sectoriales (WAINER, 1992, 2005). Hoy en día los gráficos juegan un rol importante en el análisis estadístico de datos y son una parte vital de la comunicación en ciencia y tecnología, negocios, educación, y en los medios de comunicación de masas. Con todo, diseñar gráficos para analizar y representar datos es en gran parte no científico, por ello Tufte (1997) plantea que la excelencia de los gráficos estadísticos consiste en comunicar ideas complejas con claridad, precisión y eficacia.

Por otro lado, en la literatura especializada, encontramos coincidencia en que en las graficas elementales, tales como los gráficos de barras simples o las tablas de distribución de frecuencias simples y de doble entrada (tablas de contingencia), suponen el dominio de conceptos tales como contar, escalas, origen, ejes y naturaleza de las variables. En este contexto, se asume que las personas debieran ser capaz de aplicar dichos conocimientos para decodificar, analizar e interpretar información resumida en ellos.

En otras palabras, el saber básico de la lectura y elaboración de representaciones gráficas va asociado a las habilidades de decodificar, analizar e interpretar información resumida o codificar y representar información de un fenómeno o hecho.

Si bien existen diferentes clasificaciones de dichas habilidades en la literatura especializada, en el presente estudio trabajará con la de Curcio (1989), 
toda vez que ella nos permite relacionar la habilidades propias de los procesos de comprensión lectora, es decir leer y comprender los gráficos (grafemas) representados.

El autor mencionado plantea las siguientes habilidades de lectura para comprender las gráficas o tablas de representación de información:

1.- "Leer entre los datos": lectura literal del gráfico o tabla sin interpretar la información contenida en el mismo. En otras palabras, como Habilidad asociada a la Comprensión Lectora, correspondería a la descodificación literal de la información (el dato).

2.- "Leer dentro de los datos": interpretación e integración de los datos de la tabla o gráfico; esta capacidad requiere la comparación de datos o la realización de operaciones con los datos. En otras palabras, como Habilidad asociada a la Comprensión Lectora, correspondería a la descodificación e interpretación del dato o información en su contexto.

3.- "Leer más allá de los datos": realizar predicciones e inferencias a partir de los datos sobre informaciones que no se reflejan directamente en el gráfico o tabla. En otras palabras, como Habilidad asociada a la Comprensión Lectora, correspondería a la interpretación de información implícita y explícita, así como e inferencia de ella para el mismo $\mathrm{u}$ otro contexto.

En el presente estudio se trabajó en función de las dos primeras habilidades, es decir, las habilidades de descodificación literal del dato (leer entre datos) y, aquella referida a la descodificación e interpretación del dato o información en su contexto (leer entre los datos). Dichas habilidades se evaluarán en su doble proceso: en el que implica descodifica e interpretar una información representada, como aquel que implica codificar la información en una representación distinta a la codificada en una representación gráfica inicial.

\section{DISEÑO METODOLÓGICO}

\section{Diseño del estudio}

Estudio de naturaleza mixta cuantitativo y cualitativo de tipo longitudinal descriptivo, comparativo de corte transversal. Ello dado que se pretende com- 
parar las habilidades asociadas a la lectura y elaboración de representaciones gráficas de información, presentes en profesores en ejercicio de enseñanza básica y alumnos en formación inicial docente de una universidad del centro sur del País (COOK; REICHARDT, 1986; MERLIN 1997; LATORRE et al, 1997).

\section{Población}

Alumnos en formación inicial docente de ingreso 2009 y 2010 de una universidad de Consejo de Rectores de la zona centro sur de Chile, y profesores en ejercicio en establecimientos de la provincia de Nuble que asisten a un Postitulo en Educación Matemática.

\section{Selección de la muestra}

El estudio se realizó sobre la base de una muestra seleccionada mediante un muestreo no probabilístico, del tipo estratégico o intencional; de acuerdo con Azorín y Sánchez Crespo (1986) el muestreo intencional es indicado para estudios exploratorios, para reducir costos y lograr mayor control del proceso. Se trabajó con profesores que realizan un postitulo en Educación Matemática y estudiantes universitarios de Formación Inicial Docente que asisten a la misma Universidad del Consejo de Rectores de la zona centro sur de Chile.

Específicamente, la muestra estuvo constituida por 91 individuos, de los cuales 47 eran profesores de Enseñanza Básica en ejercicio y 44 alumnos en Formación Inicial Docentes (FID) en Pedagogía en Educación General Básica de una Universidad del Centro sur del País.

\section{Instrumento}

Para la elaboración del instrumento se recurrió a la revisión los contenidos en los textos del Ministerio de Educación de Chile de primer y segundo ciclo de enseñanza básica, como también de los Mapas de Progreso del Aprendizaje, sector matemática: mapa de progreso de Datos y Azar (CHILE, 2009b), cuyo nivel de dificultad en lectura, construcción e interpretación de información no cuantitativa de una o dos variables es de nivel elemental según la escala de niveles postulada por Curcio (1989). En la siguiente tabla se muestra las especificaciones del instrumento: 
TABLA 1 - Tabla de especificaciones del instrumento

\begin{tabular}{|c|c|c|c|}
\hline Contenido & Habilidades & $\begin{array}{l}\text { Número de } \\
\text { Preguntas. }\end{array}$ & $\begin{array}{l}\text { Número de } \\
\text { Respuestas } \\
\text { Esperadas }\end{array}$ \\
\hline $\begin{array}{l}\text { Gráficos de barras } \\
\text { simples, sectoriales, } \\
\text { gráficos de barras } \\
\text { agrupadas y tablas de } \\
\text { doble entrada }\end{array}$ & $\begin{array}{l}\text { Descodificación e } \\
\text { interpretación de los datos: } \\
\text { leer entre y dentro de los } \\
\text { datos. }\end{array}$ & 6 & $\begin{array}{l}\text { Una por cada } \\
\text { pregunta } \\
\text { ( Tabla } 2 \text { y Tabla } 5 \text { ) }\end{array}$ \\
\hline $\begin{array}{l}\text { Representación gráfica } \\
\text { de datos }\end{array}$ & $\begin{array}{l}\text { Descodificación y } \\
\text { Codificación de datos } \\
\text { mediante representaciones } \\
\text { gráficas. }\end{array}$ & 3 & $\begin{array}{l}\text { Dos respuestas por } \\
\text { cada pregunta } \\
\text { (Tabla } 3 \text { ) }\end{array}$ \\
\hline $\begin{array}{l}\text { Saberes estadísticos } \\
\text { sobre representaciones } \\
\text { gráficas }\end{array}$ & $\begin{array}{l}\text { Justificación y/o Aplicación } \\
\text { de saberes estadísticos } \\
\text { para argumentar las } \\
\text { representaciones gráficas. }\end{array}$ & 4 & $\begin{array}{l}\text { Al menos una por } \\
\text { cada representación } \\
\text { ( Tabla } 5 \text { y Tabla } 7 \text { ) }\end{array}$ \\
\hline Total de Preguntas & & 13 & \\
\hline
\end{tabular}

Dicho instrumento, fue sometido a valoración de expertos cuyas aportaciones indican un índice de validez de contenido del 0,66; en el análisis de fiabilidad se obtuvo un coeficiente Alfa de Cronbach de 0,75. Ello indica que el instrumento posee una buena consistencia interna, es decir, la información es considerada fiable para los efectos del estudio.

\section{Análisis de la información}

Para el análisis de la información se consideraron estadística descriptiva univariada numérica (porcentajes, promedios), para la comparación de los porcentajes se utilizo la prueba de comparación de proporciones, según los objetivos planteados por los autores. El procesamiento de la información cuantitativa fue realizado en el paquete estadístico SPSS 13.0 y la información cualitativa en el software Qualitative Data Analysis (QDA)

\section{RESULTADOS}

Los resultados obtenidos de la aplicación del instrumento se presentan en tres secciones, en coherencia con los objetivos del estudio. En primer lugar, se entrega información sobre los porcentajes de logro asociadas a la habilidad de 
descodificación e interpretación de la información (datos) entregada en representaciones gráficas de barras y tablas; en segundo lugar, el porcentaje de logros asociadas a las habilidades de descodificación y codificación en representaciones gráficas; y finalmente, en tercer lugar, se describe y analizan los argumento que dan los diferentes actores para explicar las nuevas representaciones solicitadas (se espera que apliquen sus saberes estadísticos).

El objetivo número cuatro, que espera comparar a ambos grupos (profesores en ejercicio versus alumnos en Formación Inicial), será abordado en cada una de las tres secciones señaladas en el párrafo anterior. En otras palabras, al evaluar las habilidades de descodificación, interpretación, codificación y aplicación de saberes, paralelamente se presentarán y describirán comparativamente los datos de ambos grupos.

\section{Descodificacion e Interpretación de Información Entregada en Diversas Representaciones Gráficas: Leer Entre y Dentro de los Datos (Tipificación de las Habilidades)}

En la siguiente tabla se muestran los resultados obtenidos de la descodificación que realizan todos los encuestados de las diferentes representaciones gráficas entregadas, en general el 90,0\% realiza algún tipo de descodificación de la información, sin embargo solamente un $79,0 \%$ realiza una descodificación adecuada de la información resumida en gráficos y tablas.

Al considerar la distribución de los porcentajes de logro de los estudiantes en Formación Inicial y Profesores en ejercicio, se tiene que tanto alumnos $(40,0 \%)$ como profesores $(45,0 \%)$ presentan los porcentajes más descendidos, no presentando diferencias estadísticamente significativas, en la descodificación de un gráfico de barras agrupadas para representar la información en una tabla de distribución de frecuencia; en cambio los porcentajes de mayor logro se encuentran en la descodificación de la información presente en un gráfico de barras simples, donde los alumnos y profesores presenta porcentajes del 95,5\% y 89,3\% respectivamente, no presentándose diferencias estadísticamente significativas a un nivel de significación del 5,0\% en ambos grupos. Ello se puede deber al hecho que es una de las representaciones más usadas a nivel escolar de enseñanza básica y, por otro lado, porque hay menos información representada en un gráfico de barras simples que uno de barras agrupadas (univariada versus bivariada). 


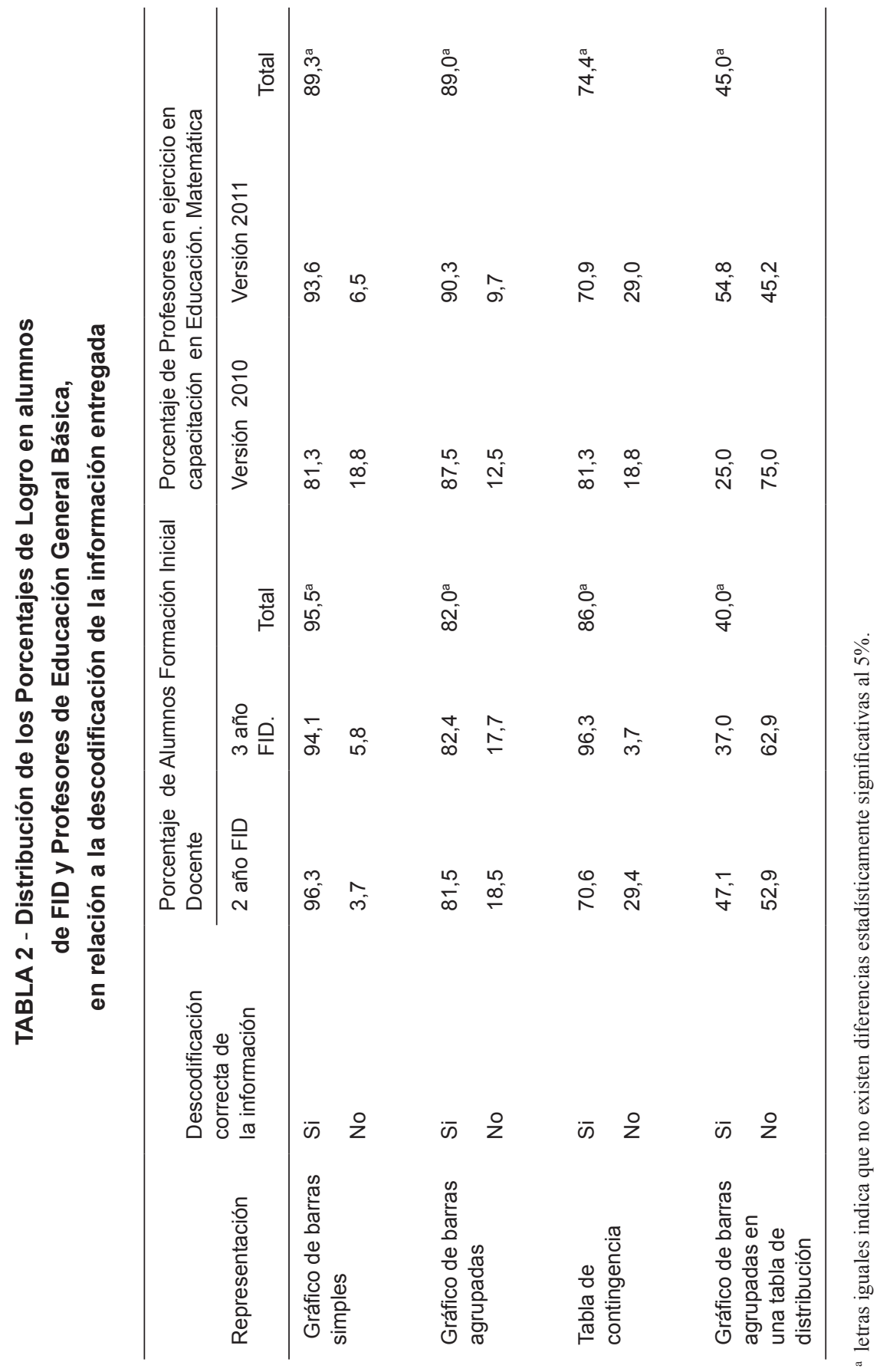


Otra tipo de representación muy usual en estadística al momento de realizar estudios comparativos de información no cuantitativa son los gráficos de barras agrupadas (rol similar juegan las tablas de doble entrada o tablas de contingencia), por ello, a los encuestados se les entrego la información sobre en que ocupan su tiempo libre estudiantes de tres diferentes colegios (Jugar, ver Televisión o Estudiar). Se les consulta sobre que podían concluir de la información entregada en la tabla (leer dentro de los datos según la clasificación de Curcio), los alumnos en FID y los profesores en ejercicios logran porcentajes promedios que fluctúan entre un $74,4 \%$ y un $89,3 \%$, no presentándose diferencias estadísticamente significativas entre dichos porcentajes promedios, lo cual nos estaría indicando que presentan competencias en la descodificación de tales representaciones gráficas.

Sin embargo, que los profesores en ejerció presenten porcentajes de logro más descendidos $(74,4 \%)$ que los alumnos de FID en la descodificación de la información proporcionada en una tabla de doble entrada, resulta preocupante, considerando que según lo explicitado en los mapas de progreso y del Decreto Ley $\mathrm{N}^{\circ} 256$, los profesores en ejercicio, deberían enseñar a codificar y decodificar información proporcionada en tablas de contingencia a los alumnos del sistema escolar.

\section{Sobre las Habilidades de Codificacion de Representaciones Gráficas}

\section{Clasificación de Habilidades de Representación Graficas}

Entre las habilidades esperadas en los alumnos de cualquier nivel de enseñanza primaria, secundaria o terciaria, es aquella referida a la elaboración de representaciones gráficas que supone el desarrollo de las habilidades de descodificación de un fenómeno, hecho, representación, entre otras, y la posterior codificación en una representación gráfica. Ello, además, supone la aplicación de los saberes estadísticos adquiridos en relación a los tipos, características, procedimientos y de representación gráfica de la información. Para tal efecto a los encuestados se les entregó tres representaciones gráficas: barras univariada, bivariada y una tabla de distribución de frecuencia bivariada (tabla de contingencia), solicitando que realizarán dos representaciones graficas distintas que permitieran resumir la información entregada (decodificar y luego codificar). En la siguiente tabla se entregan los porcentajes de logro alcanzados por los profesores en ejercicio y alumnos en FID. 


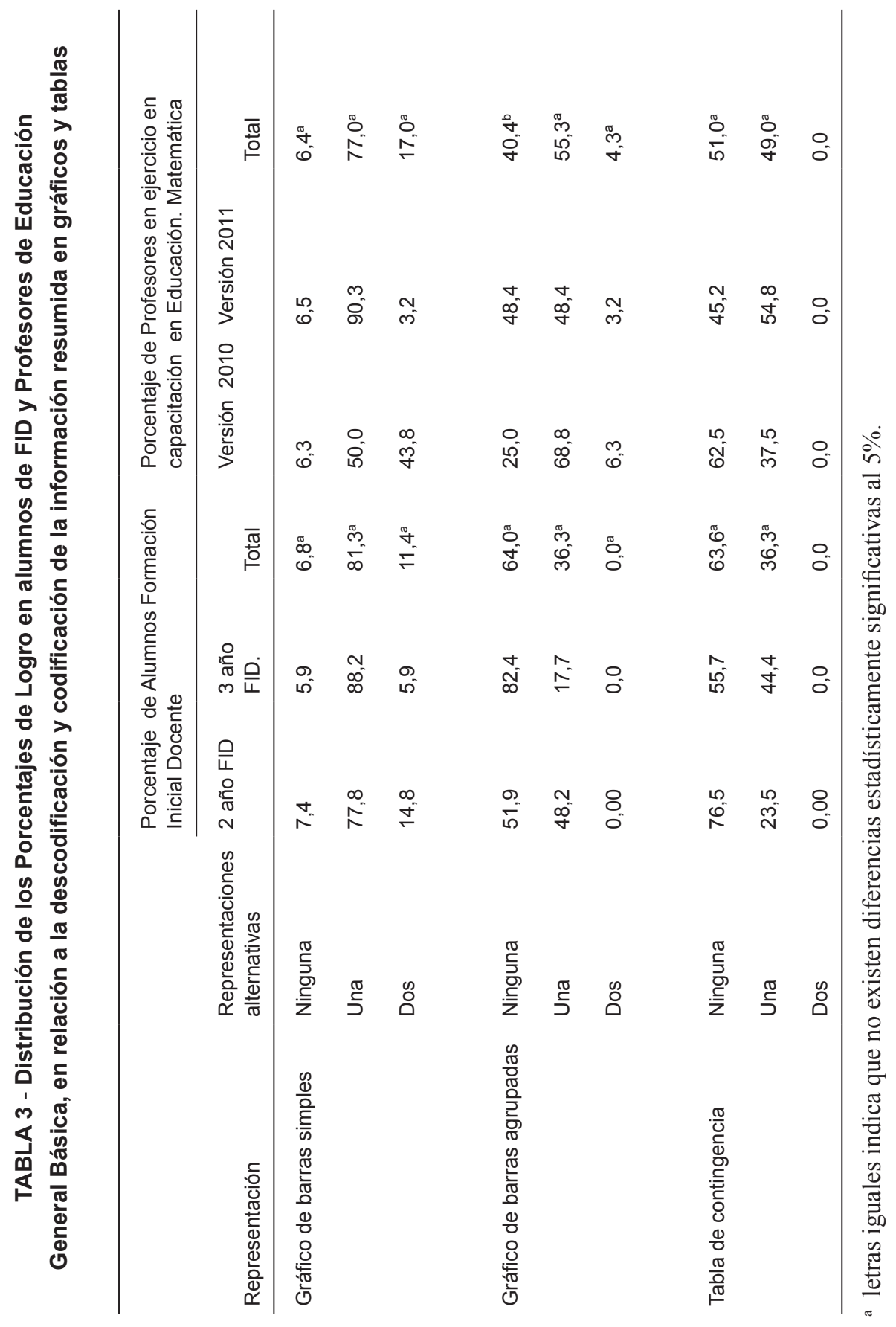


De la tabla se puede inferir, en general, que no existen diferencias significativas entre los porcentajes promedios abstenidos entre los alumnos en formación inicial docente y profesores en ejercicio. No obstante lo anterior, se puede observar que los profesores y alumnos porcentualmente realizan correctamente a lo más una representación gráfica. En particular los mayores porcentajes de logro se encuentran en la descodificación y codificación del gráfico de barras simples de los alumnos en FID (81,3\%) y los profesores en ejercicio $(77,0 \%)$. Sin embargo los encuestados presentan habilidades más descendidas en la descodificación y codificación de un gráfico de barras agrupadas (representación bivariada), donde el $64,0 \%$ de los alumnos y el $40,4 \%$ de los profesores no entregan ninguna representación alternativa, sólo el 55,3\% de los profesores en ejercicio presentan una representación alternativa a la información proporcionada, situación similar ocurre con la tabla de contingencia.

Lo anterior puede deberse, en parte, al hecho que los gráfico simples son los más usados y conocidos a nivel de enseñanza básica y media, y el brusco descenso de la habilidad en representaciones divariadas, tal vez por la complejidad asociada a la cantidad de variables o información representada (bivariada).

\section{Sobre lo Representado gráficamente}

En la siguiente tabla se muestra los diferentes tipos de gráficos que los encuestados proponen como representaciones alternativas de un gráfico de barras simple y de un gráfico de barras agrupadas.

En la tabla se puede apreciar, en el caso de un gráfico de barra simple, en promedio el $78,1 \%$ de los alumnos construye un gráfico sectorial y solamente un $31,1 \%$ de los profesores, presentándose diferencias significativas entre ambos grupos. No obstante lo anterior los profesores en ejercicio optan por entregar dos representaciones como es el caso de una tabla de frecuencia $(51,1 \%)$.

Por otro lado cuando se les solicita decodificar y codificar nuevamente la información de un gráfico de barras agrupadas los profesores y alumnos en FID optan por la misma representación gráfica aun cuando la pregunta de manera explicita dice "entregue dos formas diferentes de representar la misma información presentada en el gráfico". Las representaciones más usuales realizadas por los alumnos son el gráfico de barras simples el cual ciertamente no permite visualizar información divariada, en el caso de los profesores el 18,8\% realiza un gráfico de líneas el cual se utiliza en general para variables cuantitativas y no cualitativas. Lo anterior deja de manifiesto que tanto alumnos y profesores carecen de conceptos teóricos en relación a las representaciones gráficas. 


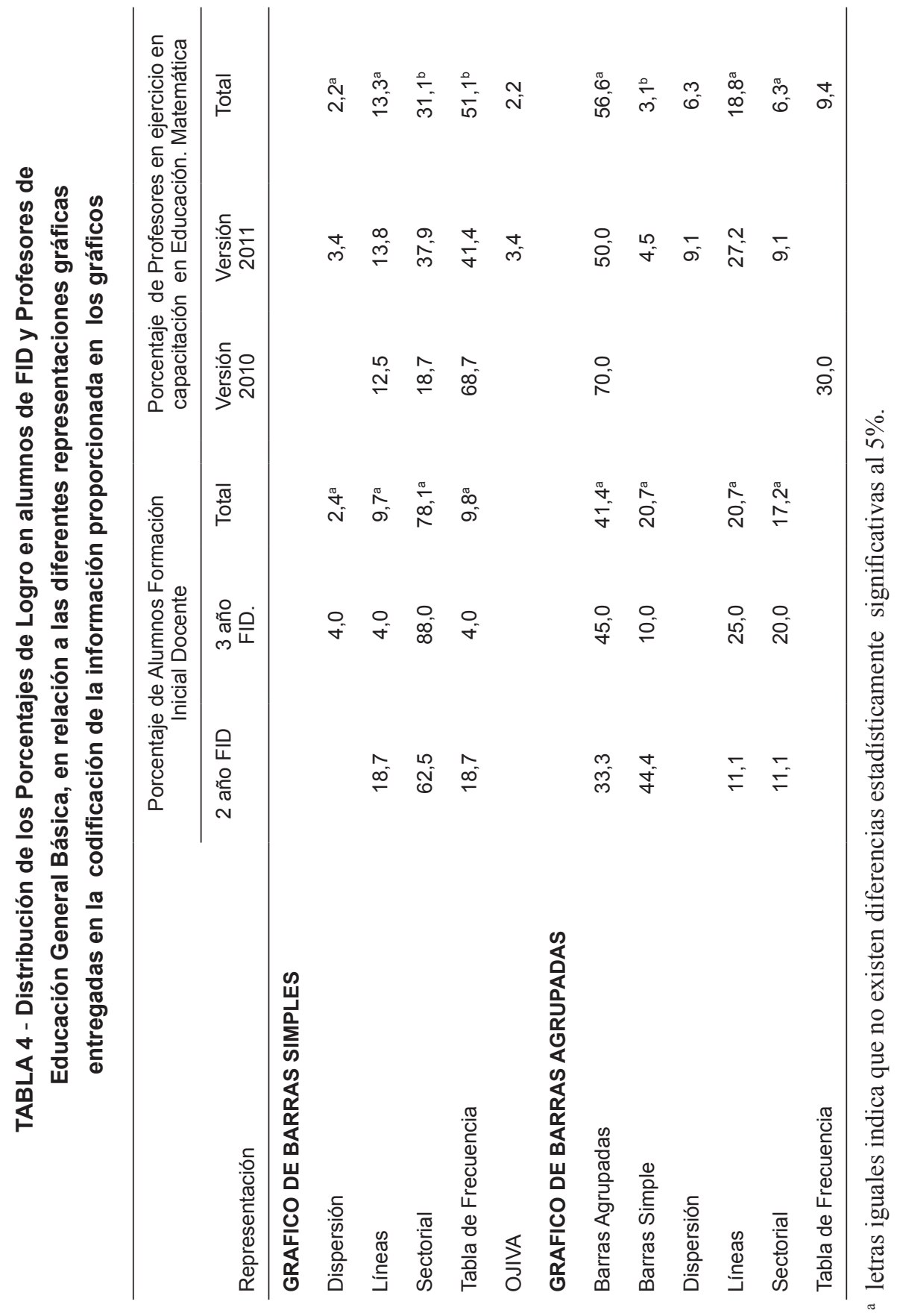




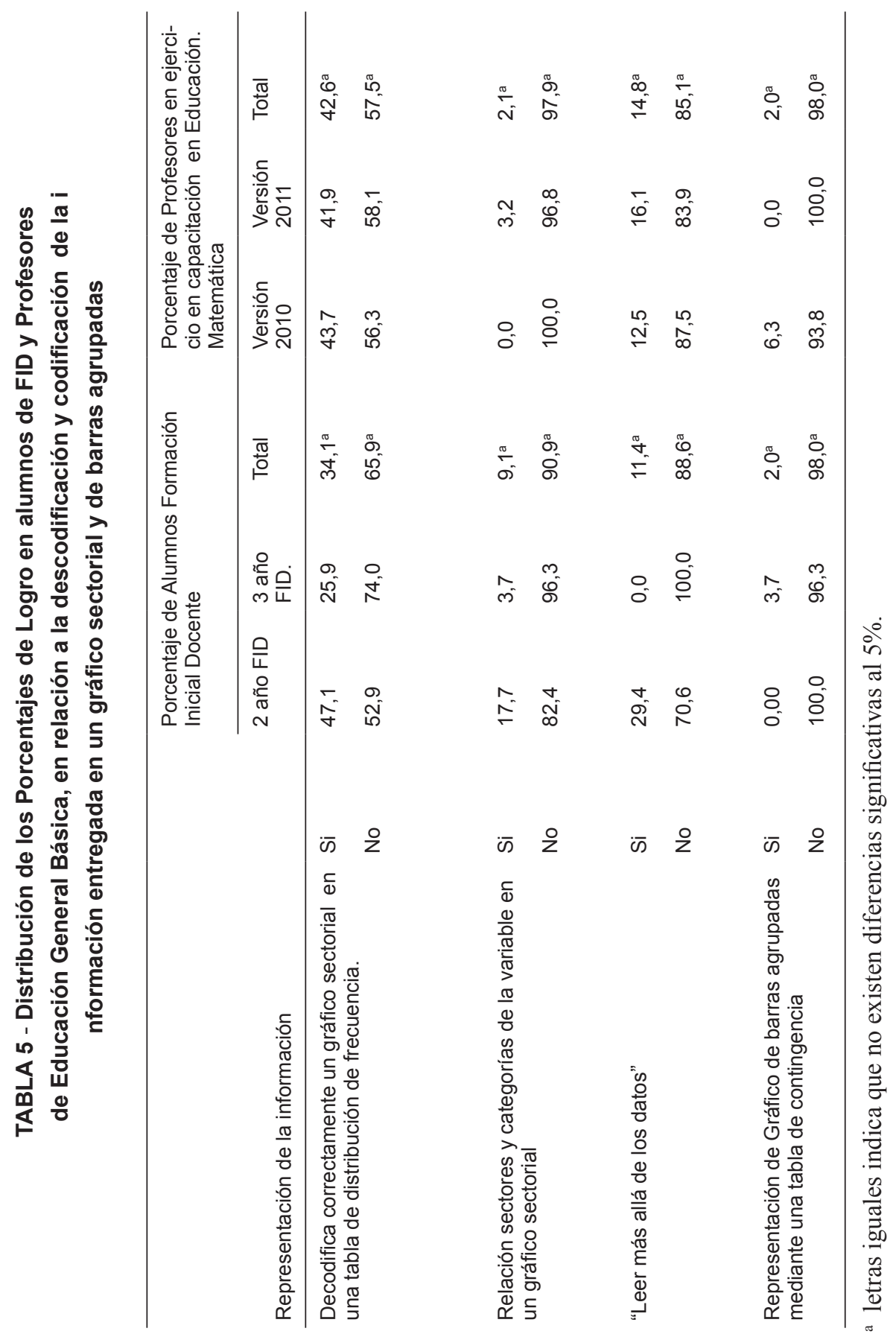


En la Tabla 5 se muestran los porcentajes de logro de la descodificación de un gráfico sectorial en una tabla de distribución de frecuencia univariada, como también su estructura y un gráfico de barras agrupadas en una tabla de distribución de bivariada.

En general, según se observa en la tabla anterior, no se presentan diferencias significativas en los promedios abstenidos por los estudiantes en formación inicial docente y profesores en ejercicio en relación a la codificación de la información entregada en un gráfico sectorial y de barras agrupadas.

No obstante lo anterior, se puede inferir que, en general, los alumnos y profesores no presentan habilidades para la descodificación de gráfico sectorial, ello porque solamente un $34,1 \%$ de los alumnos y un $42,6 \%$ de los profesores construyen una tabla de distribución de frecuencia univariada.

Por otro lado, solamente el 9,1\% de los alumnos en FID y el 2,1\% de los profesores relacionan correctamente (descodificación en contexto), las categorías de las variable presentadas en el estudio, con el peso de cada categoría del gráfico sectorial.

Finalmente, en relación a la interpretación de la información, solamente un $2,0 \%$ de los alumnos y profesores leen más allá del gráfico (análisis e interpretación), lo que resulta altamente preocupante para el sistema, toda vez que ellos deben o deberán enseñar dicha habilidad.

\section{Clasificacion de las Habilidades de Descodifiacción y codificación de Representaciones Gráficas Según Modelo de Curcio}

La siguiente tabla muestra los porcentajes de logro para leer entre y dentro de los datos (descodificación y análisis) obtenidos por los alumnos en FID y profesores en ejercicio, según la clasificación dada por Curcio (1989). En ella, se puede observar que, en general, los alumnos en FID y profesores en ejerció se encuentran en el nivel de "leer entre los datos", es decir presentan la habilidad más básica asociada a la descodificación y análisis de datos de gráficos de barras simples, agrupadas y tablas de contingencias con porcentajes que fluctúan entre un $92,9 \%$ a un $100,0 \%$ de los encuestados.

De la tabla también, se destaca que en la lectura (descodificación) de un gráfico de barras simples, tanto alumnos y profesores son clasificados en el nivel más básico de los niveles propuesto por Curcio. Ello resulta preocupante, considerando que a nivel de enseñanza básica los profesores en ejercicio y los alumnos en FID deben manipular estos objetos matemáticos no solamente al 


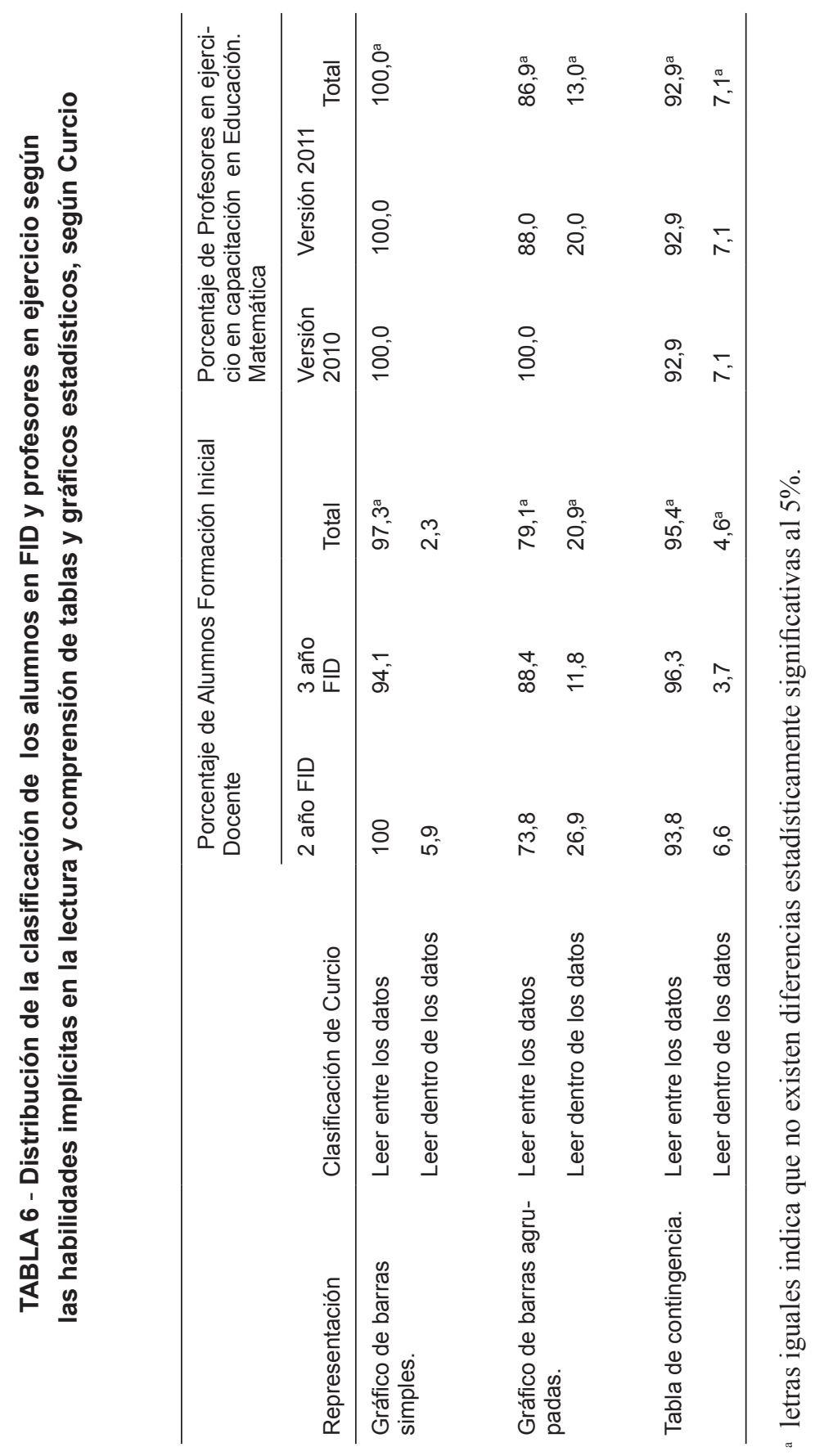


nivel de la construcción de los mismos. Si bien, no existe diferencia significativa entre los grupos, surge la interrogante de por qué los profesores presentan valores porcentuales de logro más descendidos en relación a los alumnos en FID, y si ello, a modo de pregunta, tiene relación con el hecho de que dichas habilidades y contenidos al ser relativamente nuevos en el currículo escolar, no fueron enseñados en su tiempo de formación a los actuales profesores en ejercicio.

\section{ARGUMENTOS QUE EMPLEAN LOS DIFERENTES ACTORES PARA LA ELABORACIÓN DE LAS NUEVAS REPRESENTACIONES SOLICITAS}

\section{Codificación de argumentos}

Como se señalo en el Marco Metodológico, y en coherencia con el tercer objetivo específico de nuestro estudio, dos de los reactivos del instrumento se emplearon específicamente para que argumenten o justifiquen las nuevas codificaciones representadas a partir de representaciones previas (gráfico de barra simple y agrupada).

En la representación de gráfico de barras simples se ilustraba las preferencias de los regalos que desean para navidad los alumnos de un cierto curso. Por su parte, en la representación de la tabla de contingencia se entregaba información sobre las actividades que realizan los alumnos (jugar, ver televisión, estudiar) de una comuna en sus tiempos libres de diferentes colegios.

Para el análisis de las respuestas se empleó el software Qualitative Data Analysis (QDA), siendo las unidades de análisis la respuesta dadas por los sujetos de la muestra, construyendo codificaciones y categorías a partir de ellas, por lo cual puede existir más de un argumento (codificación) en ella y, al mismo tiempo, pertenecer a más una de categoría. En otras palabras, mediante el método inductivo se codifican las respuestas para posteriormente construir las categorías semánticas (NAVARRO; DÍAZ 1994; PÉREZ, 1994).

En la Tabla 7 se entrega el conjunto de categorías semánticas construida a partir de las codificaciones, en la cuál se puede apreciar la existencia de diversidad de argumentos, no obstante, hay una clara tendencia en los dos grupos de la muestra a dar argumentos asociados a lo útil (Utilidad) de la Representación hecha por ellos, pues se encuentran los porcentajes más altos de la tabla.

En un segundo lugar, hay una variedad de argumento asociados a saberes estadísticos de distinta naturaleza (más adelante se mostrarán las codificaciones 

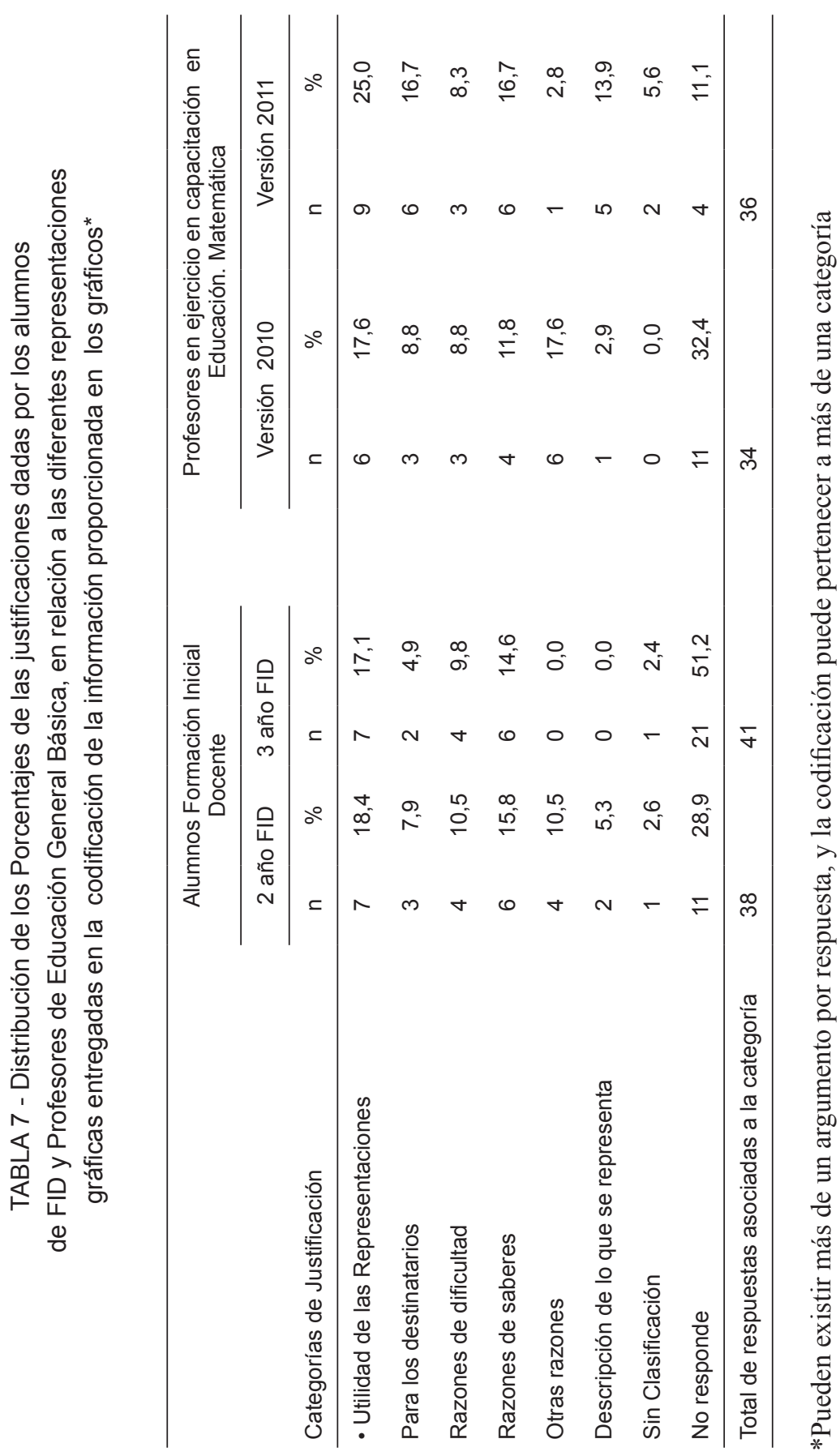
de esta categoría). Posteriormente los argumentos varían en tendencia según el grupo de la muestra.

Cabe destacar también, el alto porcentaje de No respuesta, espacialmente en los estudiantes en FID y Profesores en ejercicio. Sin embargo, es llamativo que estos porcentaje disminuyen considerablemente entre los dos grupos pertenecientes a la muestra de estudiantes, es decir, en los estudiantes con un año de estudios no responden en un 51,2\% y los con dos años de estudios a 28, 9\%; por su parte, en los profesores en ejercicio se produce el fenómeno contrario, puesto que lo que poseen dos años de capacitación el 32,4\% no responde, en cambio, los con un año sólo lo hace el 11, 1\%.

Por otro lado, al analizar el tipo de argumento (codificado) dado en cada una de las categorías mostradas en la tabla anterior, podemos señalar lo siguiente:

a) Sobre argumentos de Utilidad de la Representación:

Los argumentos dados son de diversa naturaleza: permite resumir información, para sacar conclusiones, establecer relaciones, mostrar cantidad exacta o precisa, por ser representaciones para variables cuantitativas, para analizar con más detalle, porque permite comparar, diferenciar y/o distinguir los datos, ordenar los datos o información y, finalmente, porque permite ver proporciones.

Desde el punto de vista de la estadística los gráficos permiten resumir información y mostrar relaciones (gráficos de dispersión) o realizar comparaciones gráficos de barras agrupadas o subdivididas. En tal sentido, algunos de los argumentos dados se ajustan a dichas premisas: "permite resumir información", "establecer relaciones", "porque permite comparar, diferenciar y/o distinguir los datos" u "ordenar los datos o información"

Sin embargo, el restante de las argumentaciones no se ajusta a los supuestos y conocimientos propios de las representaciones graficas por los siguientes motivos:

- Objetivización de la información datándola de una certeza absoluta: "mostrar cantidad exacta o precisa. En tal sentido, la lectura de una representación gráfica tiene un componente de subjetividad, dado que al momento de construir un gráfico la información "se pierde" y, por lo mismo, no existiría precisión absoluta.

- Tipo de variables representadas: "por ser representaciones para variables cuantitativas". Cabe señalar que la información representada en los 
gráficos del instrumento era de carácter cualitativo y no cuantitativo, ello resulta preocupante, pues como futuros profesores de enseñanza básica deben saber enseñar representaciones con información cualitativa y cuantitativa, diferenciarla y representarla de diferentes modos.

b) Sobre argumentos asociados para los Destinatarios de la Representación:

Existe una variabilidad de argumentos tales como: porque son más fáciles o didácticos de comprender por cualquier persona o niño, para estudiantes más analíticos y visuales, por qué los niños trabajan con ellos o es lo que se les enseña, más fácil para los niños o más atractivo, más concreto y atractivo, visualmente más atractivo y, por último, más fáciles de explicar a los niños.

Como se señaló anteriormente, desde el punto de vista de la estadística los gráficos permiten resumir información y mostrar relaciones (gráficos de dispersión) o realizar comparaciones en gráficos de barras agrupadas o subdivididas. Sin embargo, en segundo aspecto no menor, es que dichas representaciones permitan resumir de manera simple la información, y por lo mismo, que sea fácil lectura la representación gráfica.

Por lo anterior, el conjunto de argumentos dados bajo esta categoría resulta plausible, toda vez que en enseñanza básica las representaciones gráficas que los alumnos deben conocer con el objetivo es que sean fáciles de construir y de explicar el mensaje que transmite la imagen.

c) Sobre argumentos asociados a la Dificultad de la Representación:

Los argumentos dados son: por qué es más simple, rápida o fáciles de hacer, mucho más cómodo y claro, más fácil para procesar la información, de fácil lectura, más claro entender lo que quiero decir, más fácil de descodificar y, finalmente, fáciles de interpretar.

Los argumentos agrupados en esta categoría se pueden subdividir en dos: aquellos que aluden a la facilidad de elaborar la representación gráfica, y los que se relacionan con la facilidad de dicha representación para su lectura o comprensión para el que lo visualiza o lo interpreta.

Considerando la premisa anterior de que las representaciones gráficas permiten resumir la información para una lectura simple de los fenómenos representados, podemos señalar que resulta plausible los argumentos dados a 
favor de la segunda subdivisión: "de fácil lectura", "más claro entender lo que quiero decir", "más fácil de descodificar" y "fáciles de interpretar".

Sin embargo, aquellos argumentos asociados a la facilidad de la elaboración de la representación, ello es parcialmente cierto, en la medida de que ello no sea por sobre la naturaleza y el mensajes de la información a representar.

d) Sobre argumentos asociados a Saberes Disciplinares para la Representación:

Los argumentos dados tienes diferentes fundamentos, tales como: es la forma básica o primaria de representar datos, porque existen dos variables (independiente y dependiente), porque permite representar la información y, porque son las formas más comunes o utilizadas.

Desde el punto de vista teórico los gráficos son objetos matemáticos que permiten resumir de manera visual información cualitativa o cuantitativa, ciertamente que los gráficos entregados eran principalmente representaciones de carácter elemental, no por ello podemos esgrimir que una representación gráfica es una forma primara de representar datos, dado que la materia prima de un gráfico es esencialmente una tabla de distribución de frecuencia ya sea univariada o bivariada. Por otro lado la existencia de dos variable no siempre se puede asociar el concepto de independiente y dependiente, ello a nivel de estadística es más utilizado en los modelos de regresión y en nuestro caso, simplemente eran representaciones descriptivas de variables cualitativas.

\section{DISCUSIÓN DE LOS DATOS Y CONCLUSIONES}

El presente estudio se focalizó en las habilidades de descodificación, análisis y codificación que supone todo procesos de lectura y elaboración de representaciones graficas, tales como tablas de distribución de frecuencias univaridas o bivariadas, gráficos de barra simple o gráficos bivariados. En otras palabras, se trata de un estudio que pretende describir siguiendo los postulado de Curcio (1989), cómo leen las representaciones y que argumentos dan para elaborar representaciones gráficas.

En este Contexto, las preguntas orientadoras se resumen de la siguiente manera: $¿$ existe diferencias en el nivel de habilidades que tienen, los profesores en ejercicio y en Formación Inicial Docente, para leer (descodificar) y Representar (codificar), analizar e interpretar datos o información? y, al codificar datos o información en representaciones gráficas, ¿qué saberes (argumentos) o conocimientos emplean o aplican para ello? 
HABILIDADES DE CODIFICACION Y DESCODIFICACION DE TABLAS Y GRAFICOS ESTADISTICOS: UN ESTUDIO COMPARATIVO EN PROFESORES Y ALUMNOS DEPEDAGOGIA EN ENSEÑANZA BASICA

Las principales conclusiones y hallazgos se ordenaran, a continuación, en función de las dos grandes preguntas orientadoras señaladas:

\section{Habilidades para leer (descodificar) analizar e interpretar datos o información}

En general podemos señalar que tanto estudiantes como profesores en ejercicio, realizar procesos de descodificación (el 90,0\% de los sujetos de la muestra), a pesar que sólo el 79,0\% lo realiza de una forma adecuada, es decir, con un análisis correcto de la información. Ello podría significar, en principio, que ambos grupos poseen un desarrollo adecuado de dicha habilidad, sin embargo, al desglosar la información, podemos señalar que el tipo o complejidad de la información y/o representación afecta estos logros.

Así pues, cuándo se trata de información representada en gráfico de barras simples los alumnos y profesores presenta porcentajes elevados de descodificación con un $95,4 \%$ y $89,3 \%$ respectivamente. Sin embargo, cuando se agrega mayor información y se representa en un grafica de barras agrupadas, se tiene como resultado una baja de los porcentajes de logro de la descodificación y análisis correcto a un 40,0\% en el caso de los alumnos, y a un $45,0 \%$ en el caso de los profesores en ejercicio.

Lo anterior, explicaría en parte, porque al emplear la clasificación de Curcio (1989) la mayoría de los alumnos en FID y Profesores en ejercicio se clasifican en el nivel uno (1) "Leer entre los datos, es decir presentan la habilidad más básica asociada a la descodificación y análisis textual de los datos, con porcentajes que fluctúan aproximadamente entre un $93,0 \%$ a un $100,0 \%$ de 1 s encuestados. Sin embargo, sólo el 20,9\% de los alumnos en FID y un $13,0 \%$ de los profesores en ejercicio se pueden clasificar en la categoría de "Leer dentro de los datos" (segundo nivel de los cuatro postulados).

De lo cual, podemos inferir que dicha habilidad no está bien desarrollada y, además, estaría relacionada con la cantidad de la información y complejidad de la representación, por tanto, ello nos hace suponer que el adecuado nivel de desarrollo en la habilidades de descodificación y análisis en información encontrada en el estudio, puede deberse a la cantidad de información representada (una variable) y el tipo de representación empleada. Por lo mismo, a medida que aumenta la cantidad de información a ser representada (dos o más variables) y/o el tipo de representación (por ejemplo, gráfico de barra agrupada) disminuye el nivel de logro de dicha Habilidad. 
En resumen, podemos inferir que ambos grupos no poseen un desarrollo adecuado de la habilidad, pues al dominarla no deberían tener dificultades para leer graficas con mayor grado de dificultad en la información representada. El que no tengan gran dificultad en leer información de graficas simples puede deberse al uso generalizado de dichas representaciones en el ciclo escolar de básica en nuestro país, y no necesariamente a los conocimientos y habilidades estadísticas necesarias para leer en y dentro de las datos como lo señala Curcio (1989).

\section{Habilidades para Codificar datos o información a partir de una Representación Gráfica}

Como se señalo anteriormente, la capacidad de realizar representaciones gráficas supone habilidades de descodificar y codificar la realidad y/o fenómeno a representar, además como la capacidad de aplicar saberes estadísticos adquiridos en relación a los tipos de representación gráfica de la información. Para evaluar dichas habilidades, se les entregó información representada en gráfico de barras simples, agrupadas, y una tabla de distribución de frecuencia bivariada (tabla de contingencia), de la cuáles deberían decodificar la información para codificarla en una representación distinta a la entregada.

En general, podemos señalar que los alumnos en FID y profesores en ejercicio realizan procesos de codificación en representaciones gráficas, sin embargo, al igual que los procesos de codificación, dicha habilidad se asocian a la cantidad de información y el tipo de dificultad de la representación. Así pues, los mayores porcentajes de logro se encuentran en la codificación del gráfico de barras simples (una variable) con $81,3 \%$ de logro en los estudiantes y $77,0 \%$ en los profesores en ejercicio.

No obstante, cuando la información corresponde a dos o más variables, la habilidad de codificación descendió considerablemente, toda vez qué 64,0\% de los alumnos y el $40,4 \%$ de los profesores no entregan ninguna representación alternativa, sólo el 55,3\% de los profesores en ejercicio presentan una representación alternativa a la información proporcionada, situación similar ocurre con la tabla de contingencia.

Por lo anterior, podemos inferir que dicha habilidad no está bien desarrollada y, al igual que la habilidad de descodificación, parece que ella se relaciona con la cantidad de información representada y el tipo de representación empleada. Por lo mismo, a medida que aumenta la cantidad de información (dos o más variables) baja el nivel de logro de dicha Habilidad. 
Otro hallazgo relevante, alude al poco dominio teórico-conceptual, o la capacidad de aplicación de los mismos, al momento de realizar representaciones, dado que el $41,4 \%$ de alumnos en FID y el 56,6\% de profesores en ejercicio realizan la misma representación gráfica aun cuando se le pedía explícitamente "entregar dos formas diferentes de representar la misma información presentada en el gráfico". Sumado al hecho que las representaciones más usada por los alumnos fue el gráfico de barras simples (que se utiliza para representar información univariada y no para información bivariada que era nuestro caso); por su parte, los profesores en ejercicio, el 18,8\% realiza un gráfico de líneas el cual se utiliza, en general, para variables cuantitativas y no cualitativas que era nuestro caso.

\section{Sobre los argumentos para la representación:}

Desde el punto de vista de la estadística resulta relevante tener claridad la utilidad de la representación gráfica en general, así cómo en la particularidad de cada una de las representaciones. Es decir, que cada tipo de representación resulta más útil para un tipo de información particular que se quiere codificar como el mensaje que se quiere relevar.

En este contexto, los argumentos esgrimidos resultan plausibles y coherentes, en general, con los saberes estadísticos. Así pues, argumentos tales como "permiten resumir la información", "porque son más fáciles o didácticos de comprender por cualquier persona o niño", "de fácil lectura" y "más claro entender lo que quiero decir" entre otros.

No obstante, al relacionar los argumentos entregados con el tipo de representación gráfica elaborada, encontramos una serie de errores conceptuales que hacen dudar de los conocimientos o la aplicación de ellos por parte de los estudiantes o profesores en ejercicio. Así pues, para información de dos variables elaboran gráficos de barra simple de una variable, o para información cualitativa se argumenta que la representación hecha es por qué se trata de variables cuantitativas y cualitativas o, simplemente, porque dichas representaciones se elaboran por ser las más comunes sin ningún argumento fundado en el saber estadístico.

Finalmente, más allá de los argumentos genéricos de su utilidad y que permite resumir la información, no encontramos mayores argumentos al tipo de información a representar o la visualización rápida y autoexplicativa del mensaje a relevar con la representación gráfica. 


\section{CONCLUSIONES A MODO DE HIPÓTESIS:}

Considerando que las habilidades de descodificar y analizar la información, así como la de codificación de información, son descendidas en ambos grupos, suponemos que ello puede tener explicaciones diversas para los estudiantes en FID y los profesores en ejercicio y, por ello a modo de conclusión, postulamos dos hipótesis de trabajo inicial para ser indagadas en otros estudios:

- La Habilidad de descodificar y análisis de la información es descendida en los estudiantes en FID, puede ser explicada por el bajo desarrollo de las misma en el sistema escolar por un lado y, a el escaso desarrollo de la misma en su Formación Inicial Docente, por otro.

Dicha Hipótesis nos sugiere indagar sobre el desarrollo de dichas habilidades en sistema escolar, más precisamente, al egresar de dicho ciclo de formación y cómo estás se desarrollan y consolidan o no, en el trayecto de formación universitario propio de la Formación Inicial Docente en nuestro país.

- La Habilidad de descodificar y análisis de la información es descendida en los profesores en ejercicio, puede ser explicada, en parte, por la escasa presencia en su currículo de formación de los contenidos de estadísticas en su formación, dado que estos han adquirido importancia para el sistema escolar en la segunda mitad de década de los $90 \mathrm{y}$, por lo mismo, no fueron contenidos y habilidades enseñadas en su tiempo de formación, a los actuales profesores en ejercicio.

Asumir esta Hipótesis, nos sugiere indagar sobre el desarrollo de dichas habilidades en los profesores en ejercicio de nuestro sistema escolar, y cómo ella se relaciona con el desarrollo de las mismas en los estudiantes del sistema escolar.

Por otro lado, considerando que ambas habilidades tiende a comportarse de la misma forma en los resultados del estudio, es decir, disminuyen a medida que aumenta la cantidad de información (dos o más variables); resulta relevante indagar si hay una correlación positiva entre los niveles de logro de la habilidad de descodificación de información (con una y más variables) y los niveles de logro de la habilidad de codificación de la información (con una o más variables) en representaciones gráficas. En otras palabras, una interrogante relevante a resolver en otro estudio, es saber si existe algún tipo de correlación positiva entre ambas habilidades: descodificación de información (leer entre y dentro de los datos) y la habilidad de representación gráfica de los datos en función de la cantidad de información representada o a ser representada (una o más variables). 
Por último, considerando los resultados tanto en los alumnos en FID y los profesores en ejercicio, resulta necesario revisar los currículo de Formación Inicial Docente y las políticas de recualificación (capacitación y actualización) de los profesores en ejercicios, a objeto de mejorar sus niveles de desarrollos en dichas habilidades, toda vez que para los decretos curriculares (DECRETO LEY $\mathrm{N}^{\mathrm{o}} 256$ ), así como los mapas de progreso que orientan los sistema de medición de la calidad en nuestro país, se espera que los profesores deben enseñar a descodificar y codificar información proporcionada en tablas de distribución de frecuencias univariadas y bivariadas, como también representaciones graficas como gráficos de barras simples, agrupadas o subdivididas, a los alumnos del sistema escolar.

\section{REFERENCIAS}

ARTEAGA, Pedro et al. Las tablas y Gráficos estadísticos como objetos culturales. Números, Tenerife (Ilhas Canárias), v. 76, p. 55-67, 2010.

AZORIN, F.; SANCHEZ, J. L. Métodos y aplicaciones del muestreo. Madrid: Alianza, 1986.

BATANERO, Carmen. Didáctica de la estadística. Grupo de Investigación en Educación Estadística, Departamento de Didáctica de la Matemática. Granada: Universidad de Granada, 2001.

CHILE. Ministerio de Educacion de Chile. Objetivos fundamentales y contenidos mínimos obligatorios de la educación básica y media. Santiago: Gobierno de Chile, 2009a.

. Mapas de progreso del aprendizaje, sector matemática, mapas de progreso de datos y azar. Unidad de Curriculum y Evaluación. Santiago: Gobierno de Chile, 2009b.

COOK, T.; REICHARDT, Ch. Métodos cualitativos y cuantitativos en investigación evaluativa. Barcelona: Morata S.L., 1986.

CURCIO, F., R. Developing graph comprehension. Reston: N.C.T.M, 1989. 
GAL, I. Adult statistical literacy: Meanings, components, responsibilities.

International Statistical Review, Hague, v. 70, n. 1, p. 1-25, 2002.

HERNANDEZ, F.; SANCHO, J. Para enseñar no basta con saber la asignatura. Barcelona: Paidós, 1996.

LATORRE, A. et al. Bases metodológicas de la investigación educativa. Barcelona: Hurtado, 1997.

MERLIN, C. La investigación de la enseñanza. Barcelona: Paidós Educador/M.E.C, 1997.

MOONEY, E. S. Development of a middle school statistical thinking framework. Mathematical Thinking and Learning, Philadelphia, v. 4, n. $1,2002$.

NAVARRO, P.; DIAZ, C. Análisis de contenido. Madrid: Editorial Síntesis, 1994.

PEREZ, Gloria. Investigación cualitativa. Retos e interrogantes II : técnicas y análisis de datos. Madrid: Editorial la Muralla, 1994.

PISA. Competencias para la vida. Resultados de los estudiantes chilenos en el estudio PISA 2000. Program for International Student Assesment y Ministerio de Educación de Chile. Disponible en: $<$ http://www.simce.cl/ fileadmin/Documentos_y_archivos_SIMCE/evaluaciones_inter/pisa_2000/ informe_CHILE_PISA_2000.pdf $>$. Consultado en: 20 jun. 2009.

RODRIGUEZ, E. Tendencias en las políticas educativas latinoamericanas. Pensamiento educativo, Santiago, v. 17, n. 2, p. 133-152, 1995.

RUBILAR, Pedro R. S. et al. Evaluación de habilidades en matemática y comprensión lectora en estudiantes que ingresan a pedagogía en educación básica: un estudio comparativo en dos universidades del Consejo de Rectores. Educ. rev., Curitiba, n. 2, 2010.

TUFTE, E. The visual display of quantitatiue information. Cheshire: Graphics Press, 1997. 
WAINER, H. Understanding graphs and tables. Educational Researcher, Washington, DC, v. 21, p. 14-23, 1992.

WAINER, H. Graphical Presentation of Longitudinal Data. Encyclopedia of statistics in behavioral science. Hoboken: John Wiley \& Sons, 2005.

WILD, $C$ et al. Statistical thinking in empirical enquiry. International Statistical Review, Hague, v. 67, n. 3, 223-265, 1999.

\section{Agradecimientos}

Los autores agradecen a los evaluadores del artículo, por sus valiosos comentarios y sugerencias que han permitido mejorar la comunicación de los resultados del estudio. 